\title{
A Linear Weighted Sum Multi-objective Optimization Algorithm Based on PSO for Wideband Spectrum Sensing
}

\author{
http://dx.doi.org/10.3991/ijoe.v11i9.5058 \\ Yonghua Wang ${ }^{1}$, Yuehong $\mathrm{Li}^{2}$, Yiquan Zheng ${ }^{3}$, Ting Liang ${ }^{4}$ and Yuli Fu ${ }^{1,5}$ \\ ${ }^{1}$ South China University of Technology, Guangzhou, China \\ ${ }^{2}$ China National Electric Apparatus Research Institute, Guangzhou, China \\ ${ }^{3}$ Guangdong University of Technology, Guangzhou, China \\ ${ }^{4}$ China Electronics Technology Group Corporation No.7 Research Institute, Guangzhou, China \\ ${ }^{5}$ South-Central University for Nationalities, Wuhan, China
}

\begin{abstract}
In order to maximize throughput and minimize interference of the wideband spectrum sensing problem in OFDM cognitive radio sensor networks, a linear weighted sum multi-objective algorithm based on the Particle Swarm Optimization is proposed. The multi-objective optimization advantages of Particle Swarm Optimization are utilized to solve the optimal threshold vector of the spectrum sensing problem in OFDM cognitive radio sensor networks. So the network can get a larger throughput under the condition of small interference. The simulation results show that the proposed algorithm can make larger throughput while keeping the interference is smaller in OFDM cognitive radio sensor networks. Thus the spectrum resources are used more effectively.
\end{abstract}

Index Terms-Cognitive Radio Sensor Networks, Wideband Spectrum Sensing, Multi-objective Optimization, Particle Swarm Optimization

\section{INTRODUCTION}

With the growing demand for wireless communications, the limited spectrum resources become increasingly scarce [1-2]. In order to improve the utilization of existing spectrum and effectively solve the problem of spectrum resource scarcity, Cognitive Radio (CR) technology emerges [3-4]. Spectrum sensing [5-6] is one of the key technologies of $\mathrm{CR}$ which ensure an essential prerequisite for improving spectrum utilization. The research of spectrum sensing technology focus on cooperative spectrum sensing such as soft fusion, hard fusion and linear weighted sums fusion, etc. [7-8]. References [9-10] propose a model of simplified linear weighted sum spectrum sensing model to solve the weight based on Modified Deflection Coefficient (MDC). The performance of MDC algorithm is very close to the performance of maximum likelihood ratio method and is simpler. But MDC algorithm cannot guarantee to obtain the theoretical optimal solution. References [11-13] have introduced the intelligent optimization algorithms to get a better solution and improve the sensing performance. With the in-depth research, scholars have begun to concentrate on the wideband spectrum sensing [14-18]. Reference [16] established a multi-band joint spectrum sensing framework based on the Orthogonal Frequency Division Multiplexing (OFDM). It takes full consideration of the different condition of every orthogonal sub-channel in OFDM system. By searching the optimal threshold vector, it solves the problem of maximizing the throughput of the CR network under the condition of certain amount of interference. The simulation results verified that the algorithm based on the optimized threshold vector is more effectively for the network throughput than the traditional multi-band algorithm based on selecting the equal threshold. Based on the OFDM multi-band joint spectrum sensing framework, reference [19] used the multi-user linear weighted fusion for each sub-channel to further optimize the performance of sensing. In references [14-16, 19], the problem of the maximum throughput and minimize interference is translated into a single objective function.

Based on the optimization model of reference [16], reference [20] using a genetic algorithm to solve it. But it only considers the problem of maximizing throughput under the condition of certain system interference. In this paper, throughput and interference are considered comprehensively to study the multi-objective optimization spectrum sensing problem. A linear weighted sum multiobjective optimization model is established. And a multiobjective Particle Swarm Optimization (PSO) methods based on the linear weighted sum is proposed. By utilizing the advantages of PSO algorithm in multi-objective optimization to compute the optimal threshold vector in the OFDM CR system. The simulation results demonstrate that the proposed algorithm in this paper can find the optimum threshold vector under taking account of the throughput and interference. This method can satisfy the spectrum sensing problem of maximizing throughput while ensuring that small amount of interference.

\section{Wideband Spectrum Sensing Model ANd Multi- OBJECTIVE OPTIMIZATION}

\section{A. Spectrum sensing model}

On the basis of references [14-16], the multi-band joint spectrum sensing framework of the OFDM CR networks is shown in figure 1 . 


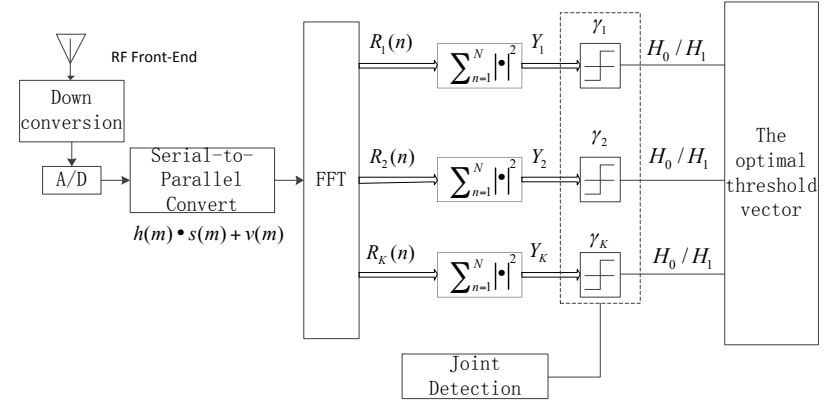

Figure 1. The multi-band joint spectrum sensing model of CR OFDM networks

This model is built on energy detection. Through the fast Fourier transform, the whole OFDM system is divided into several orthotropic sub-channels. The energy values of every sub-channel in the frequency domain are computed. Then these values are compared with a set of threshold values to judge whether the primary users (PU) exist on the sub-channels. Suppose there are K subchannels. For sub-channel $k(1 \leq k \leq K)$, the binary hypothesis model is established:

$$
\left\{\begin{array}{l}
H_{0, k}: R_{k}=V_{k} \\
H_{1, k}: R_{k}=G_{k} S_{k}+V_{k}
\end{array}\right.
$$

Where $R_{k}$ represents the complex signal in sub-channel $k$. $V_{k}$ represents the Additive White Gaussian Noise (AWGN) which obeys to normal distribution with the 0 mean and $\sigma_{v}^{2}$ variance. $S_{k}$ is the PU signal in the subchannel $k . G_{k}$ represents the channel gain between PU and Second User (SU) on the channel $k$. Generally in the detection period $G_{k}$ is considered to be constant. $H_{0, k}$ represents there exists PUs' signal on the sub-channel $k$. $H_{1, k}$ represents there is no PUs' signal on the sub-channel $k$.

The received signal of sub-channel $k$ is sampled $N$ times and the energy value in the frequency domain is computed:

$$
Y_{k}=\sum_{n=1}^{N}\left|R_{k}(n)\right|^{2}, k=1,2, \mathrm{~L}, K
$$

Suppose the threshold value of energy detection on the sub-channel is $\gamma_{k}$, then the decision rule is:

$$
\left\{\begin{array}{l}
Y_{k}<\gamma_{k}, H_{0, k} \\
Y_{k}>\gamma_{k}, H_{1, k}
\end{array}\right.
$$

According to the Central Limit Theorem, when the number of samples $N$ is large enough, the statistic $\left\{Y_{k}\right\}$ is obeyed to approximately normal distribution [16]. So $Y_{k} \sim N\left(E\left[Y_{k}\right], \operatorname{Var}\left(Y_{k}\right)\right)$, and where:

$$
\begin{gathered}
E\left[Y_{k}\right]= \begin{cases}N \sigma_{v}^{2}, \quad H_{0, k} \\
N\left(\sigma_{v}^{2}+\left|G_{k}\right|^{2}\right), & H_{1, k}\end{cases} \\
\operatorname{Var}\left(Y_{k}\right)= \begin{cases}2 N \sigma_{v}^{4}, \quad H_{0, k} \\
2 N\left(\sigma_{v}^{2}+2\left|G_{k}\right|^{2}\right) \sigma_{v}^{2}, & H_{1, k}\end{cases}
\end{gathered}
$$

According to the decision rule (3), the false-alarm probability and detection probability of sub-channel $k$ are (6) and (7) respectively:

$$
\begin{gathered}
P_{f}^{(k)}\left(\gamma_{k}\right)=\operatorname{Pr}\left(Y_{k}>\gamma_{k} / H_{0, k}\right)=Q\left(\frac{\gamma_{k}-N \sigma_{v}^{2}}{\sigma_{v}^{2} \sqrt{2 N}}\right) \\
P_{d}^{(k)}\left(\gamma_{k}\right)=\operatorname{Pr}\left(Y_{k}>\gamma_{k} / H_{1, k}\right)= \\
\\
Q\left(\frac{\gamma_{k}-N\left(\sigma_{v}^{2}+\left|G_{k}\right|^{2}\right)}{\left.\sigma_{v} \sqrt{2 N\left(\sigma_{v}^{2}+2\left|G_{k}\right|^{2}\right.}\right)}\right)
\end{gathered}
$$

Where $Q(x)=\int_{x}^{+\infty} \frac{1}{\sqrt{2 \pi}} e^{-t^{2} / 2} d t$. From the (6) and (7) we know that the selection of threshold value $\gamma_{k}$ will influence $P_{f}$ and $P_{d}$ directly. The $P_{f}$ will decrease when $\gamma_{k}$ is larger. That will make SUs have more opportunities to access spectrum hole and improve system's throughput. But it also leads the probability of missed detection $P_{m}=1-P_{d}$ increased. It will result in the increase of the amount of interference for PUs. Therefore choosing the most appropriate threshold vector $\gamma=\left[\gamma_{1}, \gamma_{2}, \mathrm{~K}, \gamma_{K}\right]^{T}$ is very important to improve the system throughput under the condition that the PUs are fully protected.

\section{B. The description of multi-objective optimization}

The total throughput of the OFDM CR system and the total amount of interference for PUs are described as (8) and (9):

$$
\begin{gathered}
R(\boldsymbol{\gamma})=\mathbf{r}^{\mathrm{T}}\left[\mathbf{1}-\mathbf{P}_{f}(\boldsymbol{\gamma})\right]=\sum_{k=1}^{K} r_{k}\left(1-P_{f}^{(k)}\left(\gamma_{k}\right)\right) \\
I(\boldsymbol{\gamma})=\mathbf{u}^{\mathrm{T}} \mathbf{P}_{m}(\boldsymbol{\gamma})=\mathbf{u}^{\mathrm{T}}\left[\mathbf{1}-\mathbf{P}_{d}(\boldsymbol{\gamma})\right]=\sum_{k=1}^{K} u_{k}\left(1-P_{d}^{(k)}\left(\gamma_{k}\right)\right)
\end{gathered}
$$

Where $\mathbf{r}=\left[r_{1}, r_{2}, \mathrm{~K}, r_{K}\right]^{\mathrm{T}}$ represent the throughput that SUs can be obtained through opportunity to use $K$ subchannels. $\mathbf{u}=\left[u_{1}, u_{2}, \mathrm{~K}, u_{K}\right]^{\mathrm{T}}$ represents the amount of interference for PUs which is caused by SUs.

Ref. [16] has studied two optimization problems of CR system. One is the optimization problem of the maximum total throughput $R(\gamma)$ of network under the condition of that the CR network satisfies the maximum interference $I(\gamma)$.The other one is the optimization of the minimum total amount of interference $I(\gamma)$ under the condition of 
the minimum throughput $R(\gamma)$. In this paper, these two indicators throughput and interference is comprehensively considered in order to solve the multi-objective optimization problem of spectrum sensing. Selecting the appropriate threshold value to obtain the maximum throughput and decrease the amount of interference. It can improve the network's communication quality and make full use of spectrum resource. Therefore the new optimization model is shown as (P1):

$$
\begin{array}{ll}
{[\max } & R(\boldsymbol{\gamma}), \min I(\boldsymbol{\gamma})] \\
\text { s.t. } & \mathbf{P}_{m}(\boldsymbol{\gamma}) \leq \boldsymbol{\alpha} \\
& \mathbf{P}_{f}(\boldsymbol{\gamma}) \leq \boldsymbol{\beta}
\end{array}
$$

Where $\boldsymbol{\alpha}=\left[\alpha_{1}, \alpha_{2} \mathrm{~L}, \alpha_{K}\right]^{\mathrm{T}}$ represents the limitation of sub-channels' interference of SUs, $\mathbf{1}-\boldsymbol{\beta}=\left[1-\beta_{1}, 1-\beta_{2} \mathrm{~L}, 1-\beta_{K}\right]^{\mathrm{T}} \quad$ represents the SUs' opportunistic access probability to the sub-channels. Therefore we only need to find the optimal threshold vector $\gamma=\left[\gamma_{1}, \gamma_{2}, \mathrm{~K}, \gamma_{K}\right]^{\mathrm{T}}$, can make the maximum throughput in $\mathrm{CR}$ networks while the interference amount is the minimum.

\section{The mathematical model of linear weighted sum multi-objective optimization}

In order to easily get the globally optimal solution of $(\mathrm{P} 1)$, the $(\mathrm{P} 1)$ is transformed into the $(\mathrm{P} 2)$ :

$$
\begin{array}{cl}
\min _{\gamma} & U=\left[f_{1}(\boldsymbol{\gamma}), f_{2}(\boldsymbol{\gamma})\right] \\
\text { s.t. } & \mathbf{P}_{m}(\boldsymbol{\gamma}) \leq \boldsymbol{\alpha} \\
& \mathbf{P}_{f}(\boldsymbol{\gamma}) \leq \boldsymbol{\beta}
\end{array}
$$

Where $f_{1}(\boldsymbol{\gamma})=R(\boldsymbol{\gamma}), f_{2}(\boldsymbol{\gamma})=I(\boldsymbol{\gamma})$. Combined with formula (8) and (9), (P2) can be transformed into a convex optimization problem satisfying some constraint conditions. As shown in (P3) and (P4):

$$
\begin{gathered}
\min _{\gamma} f_{1}(\gamma)=\sum_{k=1}^{K} r_{k} P_{f}^{(k)}\left(\gamma_{k}\right) \\
\text { s.t. } \gamma_{\min , k} \leq \gamma_{k} \leq \gamma_{\max , k}, \quad k=1,2, \mathrm{~K}, K \\
\min _{\gamma} f_{2}(\gamma)=\sum_{k=1}^{K} u_{k}\left(1-P_{d}^{(k)}\left(\gamma_{k}\right)\right) \\
\text { s.t. } \quad \gamma_{\min , k} \leq \gamma_{k} \leq \gamma_{\max , k}, \quad k=1,2, \mathrm{~K}, K
\end{gathered}
$$

In which:

$$
\begin{gathered}
\gamma_{\min , k}=\sigma_{v}^{2}\left[N+\sqrt{2 N} Q^{-1}\left(\beta_{k}\right)\right] \\
\gamma_{\max , k}=N\left(\sigma_{v}^{2}+\left|G_{k}\right|^{2}\right) \\
+\sigma_{v} \sqrt{2 N\left(\sigma_{v}^{2}+2\left|G_{k}\right|^{2}\right)} Q^{-1}\left(1-\alpha_{k}\right) \\
0 \mathrm{p} \alpha_{k} \leq 1 / 2 \text { and } 0 \mathrm{p} \beta_{k} \leq 1 / 2, k=1,2, \mathrm{~K}, K
\end{gathered}
$$

Because $f_{1}(\boldsymbol{\gamma})$ and $f_{2}(\boldsymbol{\gamma})$ are convex functions, they are suitable to use linear weighted sum method. According to the degree of sub objective function $f_{1}(\gamma)$ and $f_{2}(\gamma)$ ,we give the corresponding weighted values $a$ and $b$ ,where $a+b=1, a \mathrm{f} 0, b \mathrm{f} 0$.Then multiply each sub function with the corresponding weighted value and compute the sums, and produce the new objective function $f_{3}(\boldsymbol{\gamma})=a \cdot f_{1}(\boldsymbol{\gamma})+b \cdot f_{2}(\boldsymbol{\gamma})$. Therefor the optimal solution of $f_{3}(\gamma)$ is also the optimal solution of multi-objective function $U$. The new objective function optimization model based on (P3) and (P4) is shown as (P5):

$$
\begin{aligned}
& \min _{\gamma} f_{3}(\gamma)=a \cdot f_{1}(\gamma)+b \cdot f_{2}(\gamma) \\
& =a \sum_{k=1}^{K} r_{k} P_{f}^{(k)}\left(\gamma_{k}\right)+b \sum_{k=1}^{K} u_{k}\left(1-P_{d}^{(k)}\left(\gamma_{k}\right)\right) \\
& \text { s.t. } \quad a+b=1, \quad 0 \mathrm{p} a, b \mathrm{p} 1, \gamma_{\min , k} \leq \gamma_{k} \leq \gamma_{\max , k}, \\
& k=1,2, \mathrm{~K}, K
\end{aligned}
$$

\section{LinEar Weighted Sum Multi-OBJECTIVE OPTIMIZATION ALGORITHM BASED ON PSO}

Particle Swarm Optimization (PSO) is an evolutionary computation technology based on swarm intelligence [2123]. PSO is effective and easy to implement, less control parameters, and is widely applied to various engineering optimization filed [24-25]. In this paper, the advantages of PSO in terms of multi-objective optimization which is used to solve the multi-band joint spectrum sensing problem in CR OFDM network. It's aimed at achieving the maximum throughput and minimum amount of interference at the same time. So we propose a linear weighted sum multi-objective optimization algorithm based on PSO.

\section{A. Particle coding}

In the proposed wideband spectrum sensing algorithm based on PSO, particles use real number coding, and the scope of particles $S$. The location of each particle represents a possible threshold vector. The $i-t h$ ( $i=1,2, \mathrm{~L}, S$ ) particle's dimension is equal to the amount of wideband component channels. The $x_{i j}$ in particle's location $X_{i}=\left[x_{i 1}, x_{i 2}, \mathrm{~L}, x_{i K}\right]^{T}$ is corresponding to $\gamma_{j}(j=1,2, \mathrm{~L}, K)$. Thus the map between particles and optimization threshold vector is established. When swarm is initializing, particles are produced in a random way and the value is between $\left[\gamma_{\min , k}, \gamma_{\max , k}\right]$, where $1 \leq k \leq K$.

\section{B. Evaluation of the objective function}

In order to ensure the PSO algorithm can get the global optimal solution of (P5), the functions $f_{1}(\boldsymbol{\gamma})$ and $f_{2}(\boldsymbol{\gamma})$ should be convert into the same number lever. So (P5) will be transformed to the model as follows: 


$$
\begin{aligned}
& \min _{\gamma} f(\gamma)=a \cdot f_{1}(\gamma)+b \cdot f_{2}(\gamma)= \\
& a \cdot \frac{\sum_{k=1}^{K} r_{k} P_{f}^{(k)}\left(\gamma_{k}\right)}{\sum_{k=1}^{K} r_{k}}+b \cdot\left(1-\frac{\sum_{k=1}^{K} u_{k}\left(1-P_{d}^{(k)}\left(\gamma_{k}\right)\right)}{\sum_{k=1}^{K} u_{k}}\right) \\
& \text { s.t. } \quad a+b=1, \quad 0 \mathrm{p} a, b \mathrm{p} 1, \gamma_{\min , k} \leq \gamma_{k} \leq \gamma_{\max , k} \text {, } \\
& k=1,2, \mathrm{~K}, K
\end{aligned}
$$

Thus, for any particle $i$, the objective function is defined as $f(\gamma)=a \cdot f_{1}(\gamma)+b \cdot f_{2}(\gamma)$. The mathematical model is shown as (P6). The smaller of particle's evaluation objective function's value, the better its location. The aim is to find the particle's location where the evaluation objectives function's value is smallest, that is the globally optimal solution as $P_{g}=\left[p_{g 1}, p_{g 2}, \mathrm{~K}, p_{g K}\right]^{\mathrm{T}}$.Then the optimal threshold vector $\gamma_{o p}=\left[\gamma_{1}, \gamma_{2}, \mathrm{~K}, \gamma_{K}\right]^{T}$ will be get.

\section{Particle computation rule}

In PSO algorithm, every particle's action is determined by its spatial position $X_{i}=\left[x_{i 1}, x_{i 2}, \mathrm{~K}, x_{i K}\right]^{\mathrm{T}}$ and flight speed $V_{i}=\left[v_{i 1}, v_{i 2}, \mathrm{~K}, v_{i K}\right]^{\mathrm{T}}$.For $i-t h$ particle, the position vector $x_{i k}$ and the speed vector $v_{i k}$ of the dimension $k$ are as follows:

$$
\begin{gathered}
v_{i k}(t+1)=w v_{i k}(t)+c_{1} r_{1}\left(p_{i k}(t)-x_{i k}(t)\right)+ \\
c_{2} r_{2}\left(p_{g k}(t)-x_{i k}(t)\right) \\
x_{i k}(t+1)=x_{i k}(t)+v_{i k}(t+1)
\end{gathered}
$$

Where $t$ is the iterations number. $c_{1}$ and $c_{2}$ are learning factor, generally are random numbers between $(0,2) \cdot r_{1}$ and $r_{2}$ are the random numbers between 0 and 1 . $p_{i k}$ represents the optimal position vector in the procession of searching for particle $i . P_{g k}$ represents the globally optimal position vector in the procession of searching for swarm. $w$ represents inertia coefficient is used to control the incidence caused from current speed to the updated speed. In order to improve the search performance of algorithm, the value is computed according (15). $w_{\max }$ and $w_{\min }$ are upper and lower limit respectively. $T_{\max }$ is the maximum iteration.

$$
w(t)=w_{\max }-\left(w_{\max }-w_{\min }\right) \cdot \frac{t}{T_{\max }}
$$

\section{Algorithm Flow}

Algorithm : A linear weighted sum multi-objective optimization methods for wideband spectrum sensing based on PSO

Input: $S$ candidate solutions $X_{i}$

Output: The global optimal solution $P_{g}$
// Swarm Initialization

Step (0): Giving $S, T_{\max }, K, \gamma_{\min , k}, \gamma_{\max , k}, v_{\min , k}, v_{\max , k}$,

$$
w_{\min , k}, w_{\max , k}
$$

Step (1): $t=1, k=1, i=1$

Step (2):

$$
\begin{aligned}
& \text { For } t=1: T_{\max } \\
& \text { For } i=1: S \\
& V_{i}=v_{\min , k} * \text { ones }(1, K)+\left(v_{\max , k}-v_{\min , k}\right) * \operatorname{rand}(1, K) \\
& X_{i}=\gamma_{\min , k} * \text { ones }(1, K)+\left(\gamma_{\max , k}-\gamma_{\min , k}\right) * \operatorname{rand}(1, K)
\end{aligned}
$$

End

// According to (P6) to calculate the current value $f\left(X_{i}(t)\right)$ of the evaluation function and save the current individual $i$ optimal solution $P_{i}(t)$ and the optimal solution $P_{g}(t)$ of the Swarm.

Step (3): Evaluation Function Calculation:

$$
f\left(X_{i}(t)\right)=a \cdot f_{1}\left(X_{i}(t)\right)+b \cdot f_{2}\left(X_{i}(t)\right)
$$

Save:

$$
\begin{aligned}
& \text { fitness_pbest }(i)=\left(f\left(X_{i}(t)\right)\right), P_{i}(t)=X_{\text {pbest }}(t), \\
& \text { fitness_gbest }=\min (\text { fitness }(i)), P_{g}(t)=X_{\text {gbest }}(t)
\end{aligned}
$$

// Swarm Evolutionary computation

Step (4): Update the particle's position and velocity components according to (13), (14), (15)

For $i=1: S$

For $k=1: K$

$$
\begin{aligned}
& v_{i k}(\mathrm{t}+1)=w v_{i k}(t)+c_{1} r_{1}\left(p_{i k}(t)-x_{i k}(t)\right)+c_{2} r_{2}\left(p_{g k}(t)-x_{i k}(t)\right) \\
& x_{i k}(\mathrm{t}+1)=x_{i k}(\mathrm{t})+v_{i k}(t+1)
\end{aligned}
$$

End

Step (5): Update the particle according to step (3)

$$
\begin{aligned}
& \text { If } f\left(X_{i}(t+1)\right) \mathrm{p} f\left(X_{i}(t)\right) \\
& \quad \text { fitness_pbest }(i)=\left(f\left(X_{i}(t+1)\right)\right), \\
& P_{i}(t)=X_{\text {pbest }}(t+1)
\end{aligned}
$$

End

$$
\text { fitness_gbest }=\min (\text { fitness }(i))
$$

End

$$
P_{g}(t)=X_{\text {gbest }}(t+1)
$$

End

Step (6): $t=T_{\max }$. The algorithm terminates and outputs the global optimal solution. That is the optimal threshold vector $\gamma_{o p} \cdot P_{g}=\gamma_{o p}=\left[\gamma_{1}, \gamma_{2}, \mathrm{~K}, \gamma_{K}\right]^{T}$.

\section{SimUlation RESUlts AND ANALYSIS}

To verify the effectiveness of the proposed algorithm, the algorithm is tested by simulation. Assuming the simulation environment is the TV signal frequency OFDM system. The wideband is $48 \mathrm{MHz}$ and be divided into $K$ sub-channels averagely. Suppose the attenuation 
coefficient between PUs and SUs on the channel $k$ in the fading environment is $G_{k}$. The throughput of SUs can use in that channel is $r_{k}$. The potential interference that SUs to PUs is $u_{k}$. The parameters are shown in table 1 . In addition, the sample number of each sub-channel $N=100$ .Channel's noise power is $\sigma_{v}^{2}=1$. In order to ensure the objective function is convex function, $\beta_{k}=0.5$ and $\alpha_{k}=0.1$. Algorithm parameters are set as follows: the particle swarm size $S=100$, inertia weight $w_{\min , k}=0.4, w_{\max , k}=0.9$, learning factor $c_{1}=c_{2}=2.0$, the maximum iterations $T_{\max }=100$. The number of subchannels is between 1 and 12, the values of the system parameters $\left|\mathbf{G}_{k}\right|^{2}, \mathbf{r}(\mathrm{kbps}), \mathbf{u}$ are shown in table 1 . The results of the experiments are the average of 1,000 independent simulation results. The experiments are the performance comparison of the proposed PSO, the interior-point algorithm [16] and the GA algorithm [20].

TABLE I. SYSTEM PARAMETERS

\begin{tabular}{|c|c|c|c|c|c|c|}
\hline$k$ & 1 & 2 & 3 & 4 & 5 & 6 \\
\hline$\left|\mathbf{G}_{k}\right|^{2}$ & 0.50 & 0.30 & 0.45 & 0.65 & 0.25 & 0.60 \\
\hline $\mathbf{r}(\mathrm{kbps})$ & 612 & 524 & 623 & 139 & 451 & 409 \\
\hline $\mathbf{u}$ & 1.91 & 8.17 & 4.23 & 3.86 & 7.16 & 6.05 \\
\hline$k$ & 7 & 8 & 9 & 10 & 11 & 12 \\
\hline$\left|\mathbf{G}_{k}\right|^{2}$ & 0.40 & 0.70 & 0.35 & 0.55 & 0.75 & 0.80 \\
\hline $\mathbf{r}(\mathrm{kbps})$ & 909 & 401 & 721 & 812 & 265 & 382 \\
\hline $\mathbf{u}$ & 0.82 & 1.30 & 3.05 & 9.15 & 2.31 & 5.12 \\
\hline
\end{tabular}

\section{A. The analysis of algorithm convergence}

In Figure 2 13, the curves of the evaluation function, the total throughput, the total amount of interference with the changing of the iteration number are shown, and the influence of the different weighted value $a, b$ and the subchannel number $K$ to the method's performance are analyzed. From the simulation results we can see: with different parameters, the GA algorithm and the proposed PSO algorithm can achieve convergence and get the globally optimal solution when the number of iterations is about 50.The performances of PSO such as the minimum evaluation function, maximum throughput and minimum amount of interference are better than GA. When the subchannel number $K$ increases, the total throughput will increase, but the total amount of interference will be greater. The system will achieve different total throughput and interference under different weighted values. Therefore, choosing the appropriate weighted values according to the system environment can greatly improve the spectrum resource utilization.
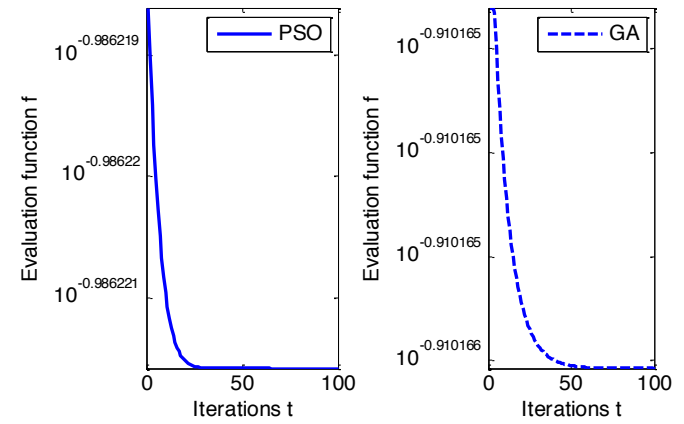

$$
\mathrm{a}=0.9, \mathrm{~b}=0.1, \mathrm{~K}=12
$$

Figure 2. Relations between the evaluation function $\mathrm{f}$ and iterations $\mathrm{t}$
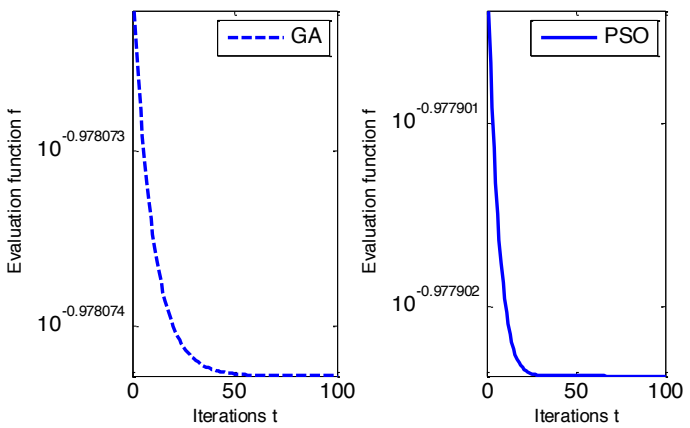

$\mathrm{a}=0.9, \mathrm{~b}=0.1, \mathrm{~K}=8$

Figure 3. Relations between the evaluation function $\mathrm{f}$ and iterations $\mathrm{t}$
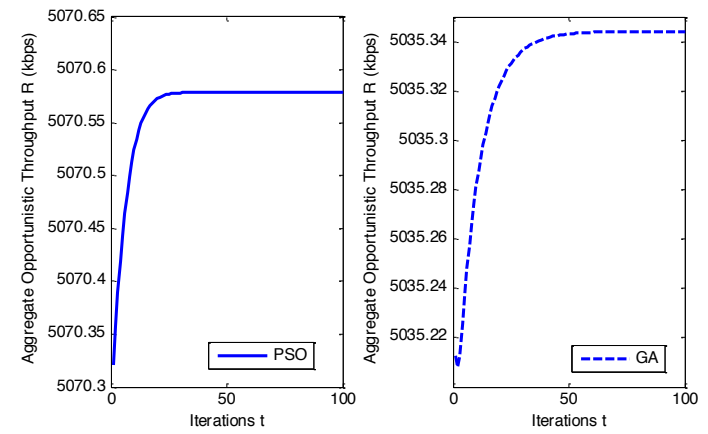

$\mathrm{a}=0.9, \mathrm{~b}=0.1, \mathrm{~K}=12$

Figure 4. Relations between aggregate opportunistic throughput $\mathrm{R}$ and iterations $\mathrm{t}$
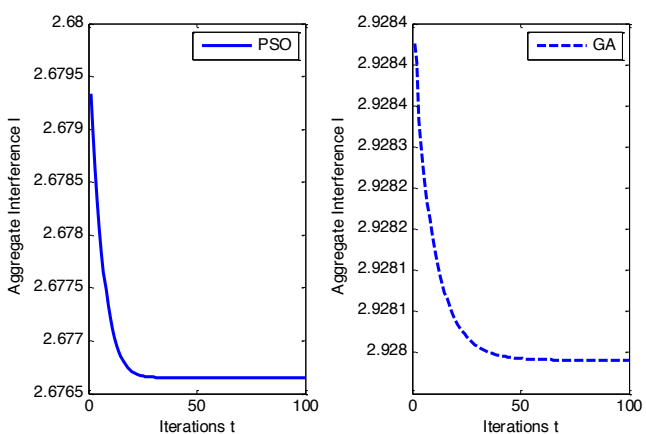

$\mathrm{a}=0.9, \mathrm{~b}=0.1, \mathrm{~K}=12$

Figure 5. Relations between aggregate interference I and iterations $\mathrm{t}$ 
PAPER

A Linear Weighted Sum Multi-ObJective Optimization Algorithm Based on PSO fOR Wideband Spectrum...

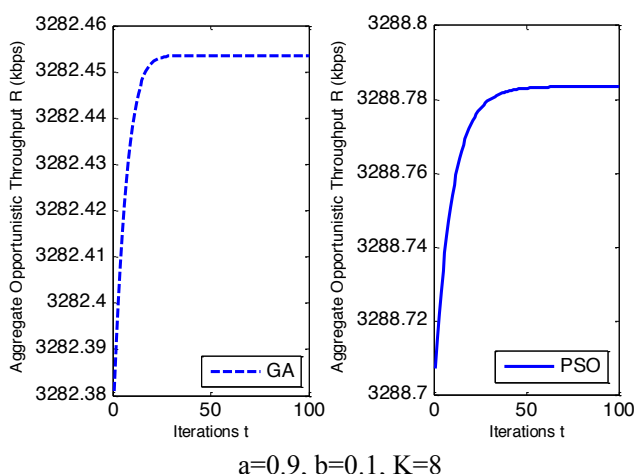

Figure 6. Relations between aggregate opportunistic throughput $\mathrm{R}$ and iterations $\mathrm{t}$
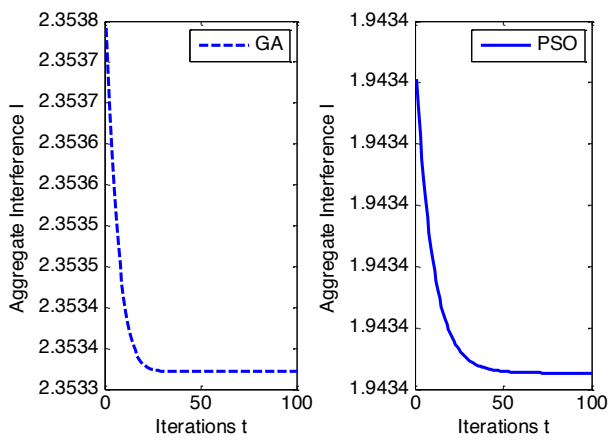

$\mathrm{a}=0.9, \mathrm{~b}=0.1, \mathrm{~K}=8$

Figure 7. Relations between aggregate interference I and iterations $\mathrm{t}$

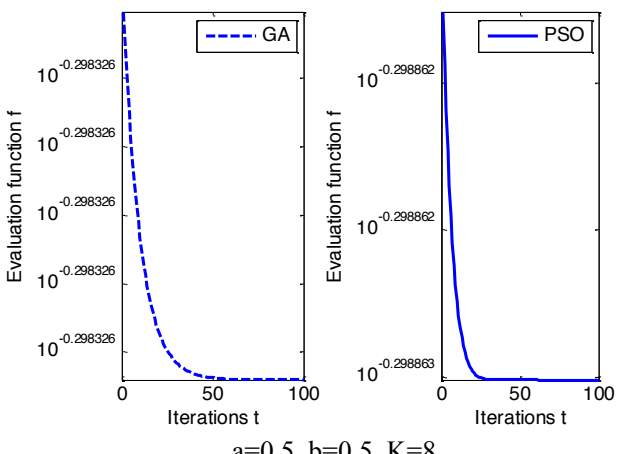

Figure 8. Relations between the evaluation function $\mathrm{f}$ and iterations $\mathrm{t}$

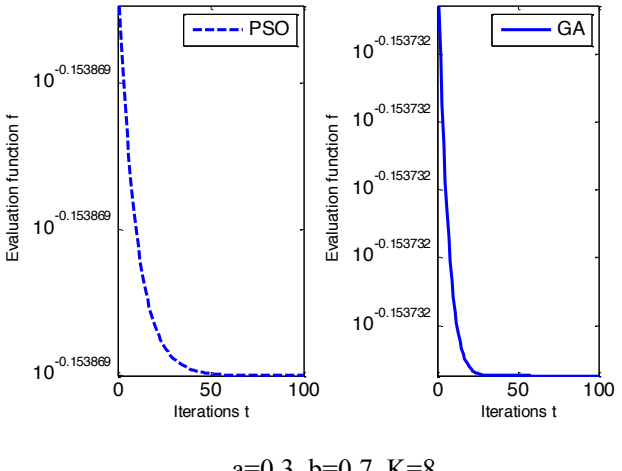

Figure 9. Relations between the evaluation function $\mathrm{f}$ and iterations $\mathrm{t}$

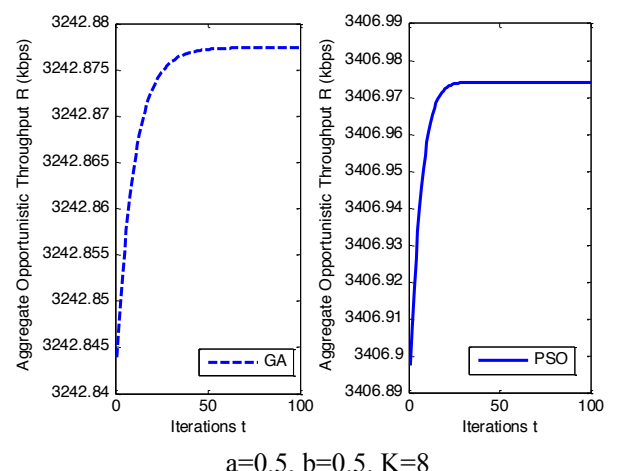

Figure 10. Relations between aggregate opportunistic throughput $\mathrm{R}$ and iterations $\mathrm{t}$
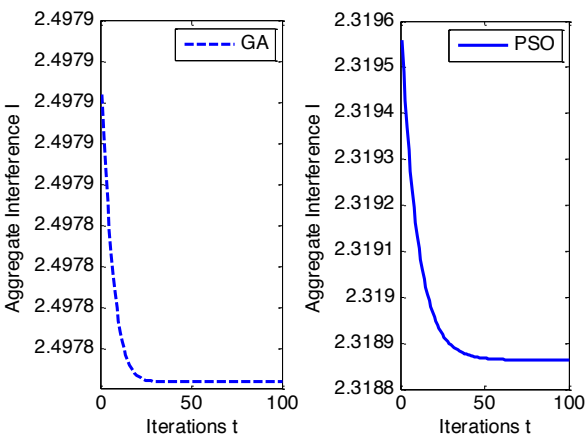

$=0.5, \mathrm{~b}=0.5, \mathrm{~K}=8$

Figure 11. Relations between aggregate interference I and iterations t

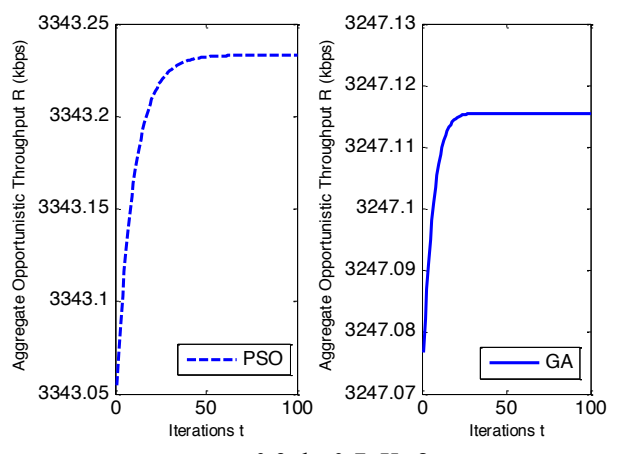

$\mathrm{a}=0.3, \mathrm{~b}=0.7, \mathrm{~K}=8$

Figure 12. Relations between aggregate opportunistic throughput $\mathrm{R}$ and iterations $\mathrm{t}$
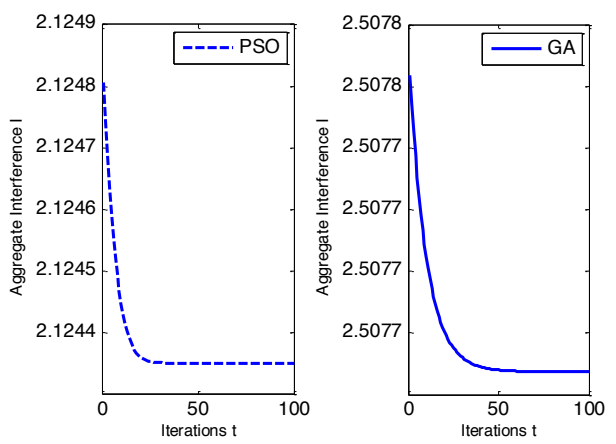

$\mathrm{a}=0.3, \mathrm{~b}=0.7, \mathrm{~K}=8$

Figure 13. Relations between aggregate interference I and iterations $t$ 


\section{B. Comparison of algorithm's performance}

Figure 14 shows the curves of the total throughput with the total amount of interference under condition of the different weights of the proposed PSO method, the interior-point method [16] and the Uniform Threshold Method. From the figure, we can see that the PSO method can achieve more throughput than the Uniform Threshold Method. And the proposed PSO method is more efficient in searching the globally optimal solution than the interior-point method.

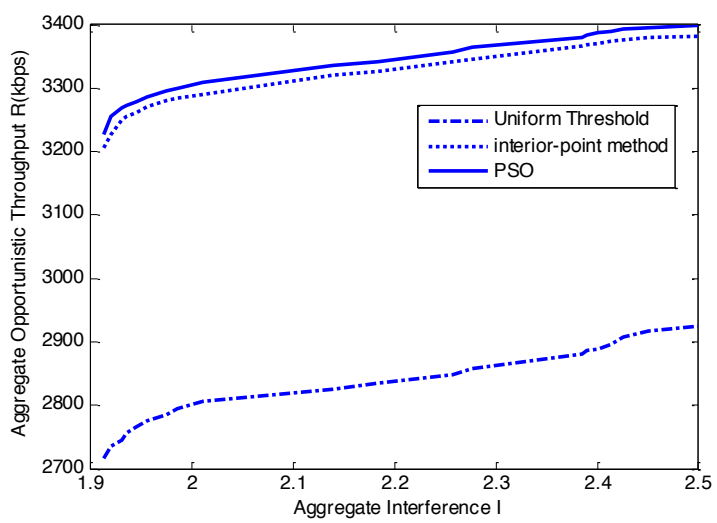

Figure 14. The comparison of the proposed algorithm with other algorithms

\section{CONCLUSIONS}

In this paper, the optimization goals of the wideband spectrum sensing problem are maximize throughput and minimize the amount of interference at the same time. And the new multi-objective optimization function is set up. A linear weighted sum multi-objective optimal optimization method based on PSO for wideband spectrum sensing is proposed. Simulation results show that the proposed method is more effectively than the genetic algorithm and traditional nonlinear programming method for solving multi-objective optimization problems. The proposed method can find the optimal threshold of OFDM wideband spectrum sensing problem, and can make larger throughput while the interference amount is low. Thus the spectrum resources are used more effectively.

\section{REFERENCES}

[1] E.Z.Tragos, S.Zeadally, A.G.Fragkiadakis, and V.A.Siris, "Spectrum Assignment in Cognitive Radio Networks: A Comprehensive Survey", IEEE Communications Surveys \& Tutorials, vol.15, no.3, pp.1108-1135, Third Quarter 2013.

[2] Lee Haeyoung;S.Vahid,; K. Moessner, "A Survey of Radio Resource Management for Spectrum Aggregation in LTEAdvanced", IEEE Communications Surveys \& Tutorials, vol.16, no.2, pp.745-760, Second Quarter 2014.

[3] G. Hattab, M.Ibnkahla, "Multiband Spectrum Access: Great Promises for Future Cognitive Radio Networks", Proceedings of the IEEE, vol.102, no.3, pp.282-306, March 2014. http://dx.doi.org/10.1109/JPROC.2014.2303977

[4] HAYKIN, "Cognitive radio: brain-empowered wireless communications", IEEE Journal on Selected Areas in Communications, vol. 23, no. 2, pp. 201-220, 2005. http://dx.doi.org/10.1109/JSAC.2004.839380

[5] K. Seshukumar, R.Saravanan,; M.S.Suraj, "Spectrum sensing review in cognitive radio," 2013 International Conference on Emerging Trends in VLSI, Embedded System, Nano Electronics and Telecommunication System (ICEVENT), pp.1,4, 7-9 Jan. 2013. http://dx.doi.org/10.1109/ICEVENT.2013.6496549

[6] A.Satheesh, S.H. Aswini,; Lekshmi, S.G.Sagar, S. M.Hareesh Kumar, "Spectrum sensing techniques A comparison between energy detector and cyclostationarity detector" ,2013 International Conference on Control Communication and Computing (ICCC), pp.388-393, 13-15 Dec. 2013. http://dx.doi.org/10.1109/ ICCC.2013.6731685

[7] F.Khan, K. Nakagawa, "Comparative study of spectrum sensing techniques in cognitive radio networks", 2013 World Congress on Computer and Information Technology (WCCIT), pp.1-8, 22-24 June 2013. http://dx.doi.org/10.1109/WCCIT.2013.6618728

[8] Jun Ma, Guodong Zhao, Ye Li, "Soft Combination and Detection for Cooperative Spectrum Sensing in Cognitive Radio Networks" ,IEEE Transactions on Wireless Communications, vol.7, no.11, pp.4502-4507, November 2008. http://dx.doi.org/10.1109/TWC.2008.070941

[9] Quan Zhi, Cui Shuguang, A.H.Sayed, "An Optimal Strategy for Cooperative Spectrum Sensing in Cognitive Radio Networks", IEEE Global Telecommunications Conference, 2007. GLOBECOM '07. , pp.2947-2951, 26-30 Nov. 2007

[10] Quan Zhi, Cui Shuguang, A.H.Sayed, "Optimal Linear Cooperation for Spectrum Sensing in Cognitive Radio Networks", IEEE Journal of Selected Topics in Signal Processing, vol.2, no.1, pp.28-40, Feb. 2008. http://dx.doi.org/10.1109/ JSTSP.2007.914882

[11] Zheng Shilian, Lou Caiyi, Yang Xiaoniu, " Cooperative spectrum sensing for cognitive radios based on a modified shuffled frog leaping algorithm", Acta Phys. Sin., vol. 59, no.5, pp.3611-3617, 2010.

[12] Wang Yonghua,Li Yuehong,Yang Jian,Wan Pin,Deng Qin, "Cooperation Spectrum sensing for cognitive radio networks based on the bacteria foraging optimization algorithm" ,International Journal of Digital Content Technology and its Applications, 6, no.4, pp.24-32, 2012.

[13] Li Xinbin, Liu Lei, "Cooperative spectrum sensing for cognitive radios based on a PA-GABC algorithm", 2011 International Conference on Electronics, Communications and Control (ICECC), pp.2604-2607, 9-11 Sept. 2011 http://dx.doi.org/10.1109/ICECC.2011.6066657

[14] Quan Zhi,Cui Shuguang, H.V.Poor, A.H.Sayed, "Collaborative wideband sensing for cognitive radios", IEEE Signal Processing Magazine, vol.25, no.6, pp.60-73, November 2008 http://dx.doi.org/10.1109/MSP.2008.929296

[15] Quan Zhi, Cui Shuguang, A.H.Sayed, H.V.Poor, "Spatial-spectral joint detection for wideband spectrum sensing in cognitive radio networks", IEEE International Conference on Acoustics, Speech and Signal Processing, 2008. ICASSP 2008., pp.2793-2796, March 31 2008-April 42008

[16] Quan Zhi,Cui Shuguang, A.H.Sayed, H.V.Poor, "Wideband Spectrum Sensing in Cognitive Radio Networks," IEEE International Conference on Communications, 2008. ICC '08. , pp.901-906, 19-23 May 2008

[17] W.Guibene, A.Hayar, M.Turki, D.Slock, , "A Complete Framework for Spectrum Sensing Based on Spectrum Change Points Detection for Wideband Signals" ,2012 IEEE 75th Vehicular Technology Conference (VTC Spring), pp.1-5, 6-9 May 2012. http://dx.doi.org/10.1109/vetecs.2012.6239955

[18] Yen Chia-Pang, Tsai Yingming, Wang Xiaodong, "Wideband Spectrum Sensing Based on Sub-Nyquist Sampling" , IEEE Transactions on Signal Processing, vol.61, no.12, pp.3028-3040, June15, 2013 http://dx.doi.org/10.1109/TSP.2013.2251342

[19] Quan Zhi, Cui Shuguang, A.H.Sayed, H.V.Poor, "Optimal Multiband Joint Detection for Spectrum Sensing in Cognitive Radio Networks" , IEEE Transactions on Signal Processing, vol.57, no.3, pp.1128-1140, March 2009 http://dx.doi.org/10.1109/ TSP.2008.2008540

[20] Deng Lilin, Zhang Cuifang, Zhou XingJian, Wen Kunhua, "Application of genetic algorithm on spectrum sensing of cognitive radio system", Application of Electronic Technology, no.3, pp.113-116, 2010.

[21] Y.V.Pehlivanoglu , "A New Particle Swarm Optimization Method Enhanced With a Periodic Mutation Strategy and Neural Networks",IEEE Transactions on Evolutionary Computation, 
vol.17, no.3, pp.436-452, June 2013 http://dx.doi.org/10.1109/ TEVC.2012.2196047

[22] Hu Mengqi, T. Wu, J.D. Weir, "An Adaptive Particle Swarm Optimization With Multiple Adaptive Methods", IEEE Transactions on Evolutionary Computation, vol.17, no.5, pp.705720, Oct. 2013 http://dx.doi.org/10.1109/TEVC.2012.2232931

[23] Wang Hu; Yen, G.G., "Adaptive Multiobjective Particle Swarm Optimization Based on Parallel Cell Coordinate System" ,IEEE Transactions on Evolutionary Computation, vol.19, no.1, pp.1-18, Feb. 2015 http://dx.doi.org/10.1109/TEVC.2013.2296151

[24] Yang Jun,Zhang Hesheng, Ling Yun, Pan Cheng,Wei Sun, "Task Allocation for Wireless Sensor Network Using Modified Binary Particle Swarm Optimization", IEEE Sensors Journal, vol.14, no.3, pp.882-892, March 2014 http://dx.doi.org/10.1109/ JSEN.2013.2290433

[25] Tao Xinmin,Liu Furong,Liu Yu,Tong Zhijing, "Multi-Scale Cooperative Mutation Particle Swarm Optimization Algorithm" ,Journal of Software, vol.23, no.7, pp.1805-1815, 2012. http://dx.doi.org/10.3724/SP.J.1001.2012.04128

\section{AUTHORS}

Yonghua Wang is with School of Electronic and Information Engineering, South China University of Technology, Guangzhou 510641, China.(e-mail: sjzwyh@163.com).
Yuehong Li is with China National Electric Apparatus Research Institute, Guangzhou 510300, China. (e-mail: 610874490@qq.com).

Yiquan Zheng is with School of Automation, Guangdong University of Technology, Guangzhou 510006, China. (e-mail: 991228678@qq.com).

Ting Liang is with China Electronics Technology Group Corporation No.7 Research Institute, Guangzhou 510310, China. (e-mail: lucky_1@tom.com)

Yuli Fu is School of Electronic and Information Engineering, South China University of Technology, Guangzhou 510641, China (e-mail: fuyuli@scut.edu.cn).

This work was supported in part by the China Postdoctoral Science Foundation (No.2014M552529, No.2015T80920), the National Natural Science Foundation of China (No.61471174), the Youth Foundation of Guangdong University of Technology (No.13QNZD006), the Higher Education Teaching Reform Project of Guangdong Province (No.GDJG20142179), Innovation and Entrepreneurship Training Programs for College Students (No.201411845145) and Higher Education Quality Project of Guangdong Province .Submitted 21 September 2015. Published as resubmitted by the authors 20 October 2015. 